te aufzeige. Ich diskutiere mit den Kolleginnen die vielfältigen Einflussmöglichkeiten der Gleichstellungsbeauftragten. Es beginnt schon damit, dass der Sprung an ein oberstes Bundesgericht neben der hohen fachlichen Qualifikation in der Regel eine R 2-Position voraussetzt. Ein solches Beförderungsamt ist jedoch gerade für Richterinnen und Staatsanwältinnen, die selbst Kinder betreuen, schwierig zu erreichen. Kinder sind der Karrierekiller Nr. 1, dies ist das Ergebnis der NRW-Studie „Frauen in Führungspositionen der Justiz“ aus dem Jahr 2011.

Die Gleichstellungsbeauftragten müssen daher bei den Beförderungschancen ansetzen. Sie müssen versuchen, ein transparentes, Chancengleichheit wahrendes Verfahren und eine geschlechtergerechte Vergabe der Sprungbrettpositionen zu erreichen. Mit Hilfe ihres Initiativrechts können sie zusätzliche Karrierewege für Richterinnen und Staatsanwältinnen mit Familienpflichten aufzeigen. Sie können Auskunft über die Beurteilungsnoten verlangen und prüfen, ob der Vergleich von beurteilten Frauen und Männern sowie Vollzeit- und Teilzeitbeschäftigten Verzerrungen aufdeckt. Sie dürfen Richterinnen und Staatsanwältinnen über die Karrieremöglichkeiten informieren und sie bei Bewerbungen unterstützen. Eine erfolgreiche Abordnung an ein oberstes Bundesgericht als wissenschaftliche Mitarbeiterin erhöht die Chance, als Bundesrichterin dorthin zurückzukehren. Schließlich sollten die Gleichstellungsbeauftragten einfordern, an den Entscheidungen beteiligt zu werden, wer auf die internen Vorschlagslisten aufgenommen und wer letztendlich vorgeschlagen wird. Die Gleichstellungsbeauftragten gewinnen zudem umso mehr an Einfluss, je mehr sie sich mit ihren Kolleginnen in den verschiedenen Instanzen vernetzen und regelmäßig fachlich austauschen.

Auf Bundesebene hat sich ein Arbeitskreis der Gleichstellungsbeauftragten der Bundesgerichte etabliert, der auch die
Kolleginnen des Bundesverfassungsgerichts, der Bundesanwaltschaft, des Bundesministeriums der Justiz und des Bundesministeriums für Arbeit und Soziales, das für die Wahlen zum BAG und BSG zuständig ist, einschließt. Ein wichtiges Thema ist, die Beteiligung der Gleichstellungsbeauftragten an den Sitzungen des Präsidialrats zu erreichen, bei dem sich die Kandidatinnen und Kandidaten für die Richterwahlen vorstellen müssen.

Neben der Neuen Richtervereinigung (NRV), die unsere Initiative „Frauen in die Roten Roben“ von Anfang an unterstützt, hat sich inzwischen der Bund der Richterinnen und Richter, Staatsanwältinnen und Staatsanwälte (DRB) des Themas angenommen und eine Arbeitsgruppe gebildet. Die fehlende Transparenz der Bundesrichterwahlen benachteiligt ja auch potentielle männliche Kandidaten. Unser Ansatzpunkt ist jedoch ein anderer: Wir brauchen auf dem gesamten Weg in Führungspositionen der Justiz Chancengleichheit für Frauen. Das über Jahrzehnte gepflegte Argument, es gebe nicht genügend qualifizierte Frauen, ist ausgeräumt. Betrachten wir nur die nachwachsende Frauenpower auf der einen und die geringe Zahl an jährlich zu besetzenden Führungsstellen auf der anderen Seite. Gab es 2011 nur drei Frauen auf der Wahlliste für den Bundesgerichtshof, hat man in diesem Jahr 19 Kandidatinnen präsentieren können. Die Auffassung „Wer suchet, der findet“ vertritt der djb auch im ähnlich gelagerten Projekt „Frauen in die Aufsichtsräte“. Unsere beiden Initiativen beginnen den Blickwinkel in Politik und Gesellschaft dahin zu verändern, dass Gleichstellung eben nicht von selbst kommt. Artikel 3 Absatz 2 des Grundgesetzes weist die staatlichen Repräsentanten seit annähernd 20 Jahren daraufhin, dass sie die Gleichberechtigung von Frauen und Männern tatsächlich durchsetzen müssen.

\title{
Werden Beamtinnen bei der Beurteilung benachteiligt?
}

\section{Dr. Karin Tondorf}

Mitglied der Kommission Arbeits-, Gleichstellungs- und Wirtschaftsrecht des djb; Freie Wissenschaftlerin/Beraterin, Seddiner See

Will eine Beamtin im öffentlichen Dienst aufsteigen, muss sie zu den Besten gehören. Ihre Chancen stehen gut, wenn sie eine Beurteilung vorweisen kann, die ihr eine sehr gute, wenn nicht gar eine hervorragende Leistung und eine entsprechende Eignung und Befähigung für die zukünftige Stelle bescheinigt. Doch wie verhält es sich mit der Chancengleichheit von männlichen und weiblichen Beamten, wenn es um die Beurteilung und Beförderung geht? Inwieweit können Beamtinnen (und Beamte) darauf vertrauen, dass ihre Leistungen angemessen und diskriminierungsfrei beurteilt werden?

Diese Frage stellten sich die Wissenschaftlerinnen Andrea Jochmann-Döll und Karin Tondorf ${ }^{1}$ bezogen auf den Polizeivollzugsdienst in Deutschland. Dieser Bereich ist bis heute eine Beschäftigungsdomäne von Männern geblieben, obwohl immer mehr Frauen bei der Kriminal-, Schutz- und Bereitschaftspolizei ihren Dienst tun. Seit geraumer Zeit hegen Frauen in der Gewerkschaft der Polizei (GdP) sowie Gleichstellungsbeauftragte den Verdacht, dass Frauen und Teilzeitbeschäftigte bei der Beurteilung und Beförderung benachteiligt sein könnten. Es gab jedoch nur wenige aussagekräftige Beurteilungsstatistiken, auf die sie sich berufen konnten.

Mit der neuen wissenschaftlichen Untersuchung liegen nun umfangreiche Daten über Beurteilungsergebnisse von Frauen und Männern im Polizeivollzugsdienst vor: 12 Bundesländer und die Bundespolizei übersandten den Wissenschaftlerinnen ihre statistischen Beurteilungsdaten sowie die jeweils geltenden Beurteilungsrichtlinien zur Analyse. Darüber hinaus stellten sich betriebliche Expert/inn/en für Interviews zur Verfügung.

1 Jochmann-Döll, Andrea/Tondorf, Karin (2013): Nach Leistung, Eignung und Befähigung? Beurteilung von Frauen und Männern im Polizeivollzugsdienst, gefördert von der Hans-Böckler-Stiftung, Arbeitsheft 276, Düsseldorf. 


\section{Statistische „Schieflagen“ ...}

Die ausgewerteten statistischen Daten sind ernüchternd: Frauen schneiden bei der Beurteilung auffallend oft schlechter $a b$ als Männer. Diese Grundtendenz lässt sich nicht nur anhand der vergebenen Durchschnittsnoten belegen. Auch wenn die Verteilung der Bestnoten an Frauen und Männer betrachtet wird - jeweils bezogen auf ihren Beschäftigtenanteil -, sind es die Frauen, die diese Note seltener erhielten als Männer. Um ein Beispiel zu geben: Bei der Bundespolizei wurde die beste Notenstufe an 8,01 Prozent der Frauen und an 14,23 Prozent der Männer vergeben (2010). Dort erhielten nur 5,9 Prozent der teilzeitbeschäftigten Frauen diese Stufe, hingegen 11,88 Prozent der teilzeitbeschäftigten Männer.

Abweichungen von diesem Grundmuster finden sich in einigen Bundesländern, und zwar vor allem in den unteren Besoldungsgruppen, in denen Frauen stärker vertreten sind. Auch die Teilzeitbeschäftigten erhielten mitunter bessere Beurteilungen als die Vollzeitbeschäftigten. Solche partiell festzustellenden Ergebnisse zeigen, dass schlechtere Beurteilungen von Frauen und Teilzeitbeschäftigten kein „Naturgesetz“ sind, sondern ausgewogenere Beurteilungsergebnisse unter bestimmten Bedingungen durchaus erreichbar sind.

\section{... und mögliche Ursachen}

Warum erhalten Frauen im Polizeivollzugsdienst mehrheitlich schlechtere Beurteilungen als Männer? Betriebliche Expert/ inn/en der Bundespolizei sowie der Bundesländer BadenWürttemberg und Sachsen-Anhalt (Personalverantwortliche, Leitungen von Beurteilerkonferenzen, Erstbeurteiler, Personalräte, Gleichstellungsbeauftragte) nahmen zu den statistischen Befunden Stellung und erklärten die ungünstigeren Beurteilungsergebnisse von Frauen mit vielfältigen Mechanismen: So wird bei den Leistungserwartungen vielfach noch immer vom Prototyp eines männlichen, vollzeitbeschäftigten und flexiblen Polizeibeamten ausgegangen, was Beschäftigten mit geringerer Flexibilität und Präsenz - meist Frauen mit Familienpflichten und Teilzeitbeschäftigte - häufig als Leistungseinschränkung ausgelegt wird und ihnen dann Minuspunkte bei der Beurteilung bringt.

Offenbar ist es ein verbreitetes Anliegen, ein hohes Dienstalter und die damit verbundene „Lebensleistung“ mittels einer guten Beurteilung anzuerkennen, auch wenn die aktuell gezeigte Leistung dem nicht entspricht. Da die älteren Jahrgänge überwiegend männlich sind, zählen jüngere Frauen seltener zu den Bestbeurteilten. Es kommt in der Praxis mitunter vor, dass leistungsstarke Frauen auf den verdienten Aufstieg verzichten und den Vorrang einem älteren Kollegen einräumen müssen, weil er noch rechtzeitig vor der Pension ins Endamt befördert werden soll. Berichtet wurde auch über eine Beurteilungspraxis, wonach die überwiegend männlichen Beurteilenden von vornherein ein subjektives Ranking der Beamtinnen und Beamten im Kopf haben, das sich bei der Notenvergabe bestätigen soll. Einen Überblick über diese und andere leistungsfremde Faktoren, die in die Beurteilung einfließen, bietet Abbildung 1. In den Interviews kam jedoch auch zum Aus- druck, dass die Beurteilungsnote bei der Beförderung nicht immer den Ausschlag gibt: Manchmal werden auch männliche Beamte mit schlechteren Beurteilungen befördert, weil sie ein gewisses Dienstalter erreicht haben.

Inwieweit tragen - neben der Beurteilungspraxis - auch die Beurteilungsrichtlinien zu ungünstigeren Beurteilungen von Frauen bei? Festzuhalten ist, dass die meisten Richtlinien den Beurteilenden beträchtliche subjektive Beurteilungsspielräume einräumen, durch die leistungsfremde Faktoren wie soziale Aspekte, Vorurteile, Geschlechterstereotype und handfeste Interessenlagen in die Beurteilung einfließen können. Zu beurteilen sind vielfach persönliche Eigenschaften, Charaktermerkmale und Verhaltensweisen von Beamtinnen und Beamten (z.B. Zuverlässigkeit, Belastbarkeit, Umgang mit Konfliktsituationen), die zum Teil nicht eindeutig ausgelegt sind. Problematisch ist insbesondere, dass viele Leistungsmerkmale von der Wahrnehmung und Urteilsbildung der (überwiegend männlichen) Vorgesetzten abhängig gemacht werden. Diese erfolgen - so Befunde der Beurteilungsforschung - zwangsläufig selektiv und werden durch Schemata gefiltert, etwa durch geschlechterbezogene Leistungserwartungen und vorgefasste Meinungen. Zahlreiche Richtlinien verpflichten die Behörden außerdem zur Einhaltung von Richtwerten zur Verteilung der Noten (z.B. nicht mehr als 30\% sehr gut und gut), was zu Problemen führt, wenn es mehr leistungsstarke Beamt/inn/e/n gibt als vorgesehen. In diesen Fällen muss eine Auswahlentscheidung getroffen werden, wer „herausfällt“. Dabei können Aspekte wie Teilzeitarbeit, geringere zeitliche Flexibilität oder geringeres Dienstalter durchaus eine Rolle spielen und zu Ungunsten von Frauen wirken. Insgesamt kann festgehal-

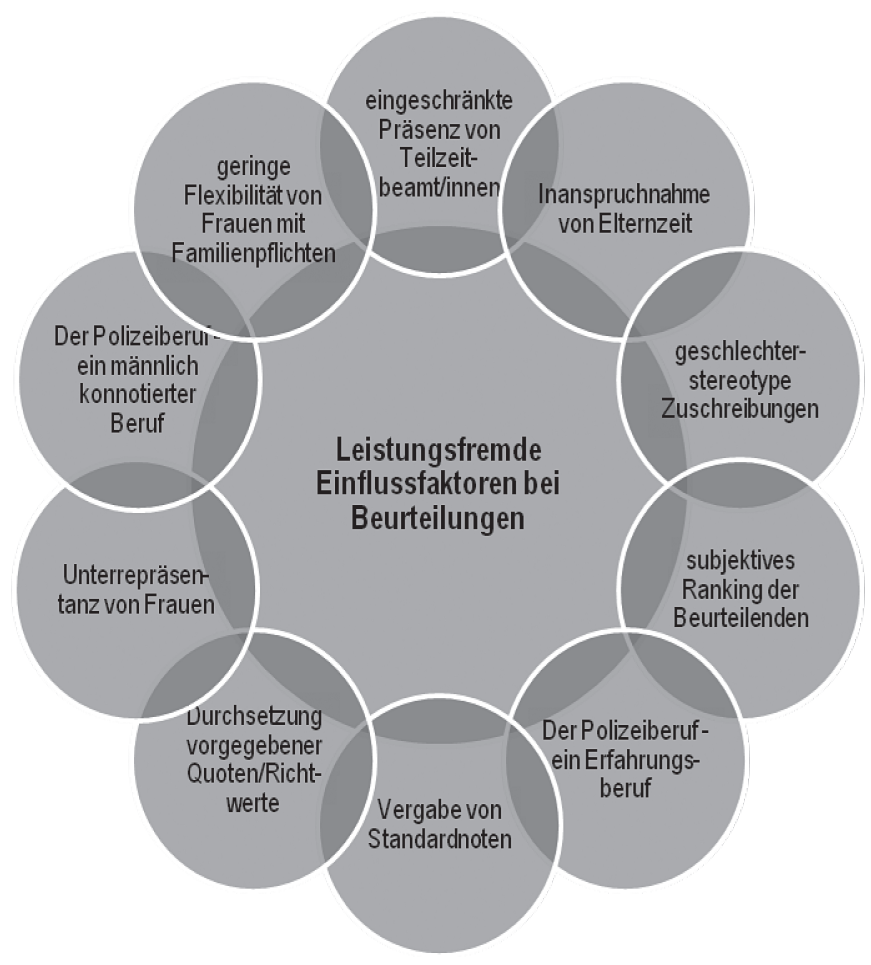

A Abb. 1: Leistungsfremde Einflussfaktoren bei Beurteilungen 
ten werden, dass vielfach Regelungen fehlen, die einer Benachteiligung aufgrund des Geschlechts vorbeugen und mit denen die Beurteilungspraxis transparenter, objektiver und diskriminierungsfreier gestaltet werden könnte.

\section{Handlungsansätze}

Aus den Analysen ergeben sich vielfältige Handlungsansätze für geschlechtergerechtere Beurteilungen, sowohl auf der betrieblichen als auch auf der Regelungsebene. Vorrangig erscheinen Maßnahmen, die die subjektiven Beurteilungsspielräume einschränken, zum Beispiel durch stärker objektivier- bare Merkmale bzw. Ziele sowie Vorgaben zu regelmäßigen statistischen Auswertungen der Beurteilungspraxis und zu ihrer Evaluation. Notwendig wären außerdem Fortbildungen für Beurteilende, die für potenzielle geschlechterbezogene Benachteiligungen sensibilisieren. Ein Verzicht auf Quoten könnte das Potenzial für Benachteiligungen weiter reduzieren. Und nicht zuletzt könnte eine stärkere Beteiligung der Gleichstellungsbeauftragten bei der Gestaltung des Beurteilungsverfahrens und -prozesses zu mehr Transparenz und einer effektiveren Prüfung der Geschlechtergerechtigkeit bei der Beurteilung führen.

\section{$\S 177$ StGB - Kritik und Verbesserungsvorschläge im Vergleich mit den Regelungen in Norwegen, Schweden und England/Wales'}

\section{Sabine Kräuter-Stockton}

Mitglied der Kommission Strafrecht des djb; Oberstaatsanwältin, Saarbrücken

Verbrechen der Sexuellen Nötigung und Vergewaltigung sind weiter verbreitet, als in der Öffentlichkeit gemeinhin angenommen. 13 Prozent der Frauen zwischen 16 und 85 Jahren, das heißt fast jede siebte, hat schon einmal sexualisierte Gewalt erlebt. ${ }^{2}$ Weil das Erleben von sexualisierter Gewalt in der Regel nicht nur langfristige soziale und psychosoziale Folgen wie Scheidung, Wohnungswechsel und Therapie für die Betroffenen nach sich zieht, sondern auch in erheblichem Maße die Gesundheit der Opfer beeinträchtigt ${ }^{3}$, kann ein moderner Staat sowohl aus Menschenrechtsgesichtspunkten als auch aus rein wirtschaftlichen Erwägungen die Augen vor dem Problem nicht verschließen.

Was unternimmt unser Staat dagegen? Als Staatsanwältin sehe ich vor meinem beruflichen Hintergrund das Strafrecht als eine wichtige Stellschraube an. Mit dem Strafrecht verfolgt der Staat, obwohl es primär um die nachträgliche Sanktionierung bereits geschehenen Unrechts geht, seit jeher auch Präventionszwecke. Würden beispielsweise keine Geschwindigkeitsüberschreitungen oder Rotlichtverstöße im Straßenverkehr mehr verfolgt, würde sich wohl kaum jemand noch an die Vorschriften halten. In diesem Sinne ist von einer möglichst effektiven Strafverfolgung auch bei Sexualverbrechen eine general- und spezialpräventive Wirkung zu erwarten. Wenn Täter damit rechnen müssen, angeklagt und streng bestraft zu werden, so wird dies zumindest einen Teil der Täter abschrecken. Wenn sie sich andererseits eine gute Chance ausrechnen können, nicht sanktioniert zu werden, sinkt die Schwelle für die Begehung von Straftaten.

Derzeit sieht die Statistik aber - nicht nur deutschlandweit, sondern europa- und wahrscheinlich weltweit - traurig aus: Nur etwa 12,7 Prozent der im Jahr 2011 in Deutschland begangenen aufgeklärten Sexualverbrechen wurden mit einer Verurteilung des Täters abgeschlossen. ${ }^{4}$ Genaue Zahlen sind nicht festzustellen, denn weil sich gerade Strafverfahren wegen Sexualverbrechen oft über mehrere Jahre hinziehen, liegen
Anzeige und Verurteilung oder Verfahrenseinstellung selten im selben Jahr. Dennoch zeigt sich deutlich, dass Sexualverbrechen im Verhältnis besonders selten abgeurteilt werden: Im Durchschnitt aller Straftaten wurde im Jahr 2011 immerhin bei etwa 24,6 Prozent der aufgeklärten Straftaten, also doppelt so häufig wie bei Sexualdelikten, der Täter verurteilt. ${ }^{5}$

Nun ist es aber nicht etwa so, dass wegen Sexualdelikten deutlich mehr Falschanzeigen erstattet würden als im Durchschnitt der Verfahren. Für den verhältnismäßig geringen Anteil von Verurteilungen gibt es verschiedene Ursachen. Neben Geschlechterstereotypen und unzutreffenden Vorurteilen bei den professionellen Akteuren liegt es sicherlich auch daran, dass bei Sexualdelikten der Nachweis der Tat, die klassischerweise nicht vor Dritten begangen wird, praktisch ausschließlich von der Aussage des Opfers abhängt: Wenn das Opfer aus gleich welchen Gründen keine Aussage mehr machen will, ist ein Nachweis in der Regel nicht möglich. Dann gibt es auch immer wieder Fälle, in denen deutlich wird, dass unser Hand-

1 Vortrag, gehalten am 8.2.2013 auf der Tagung „Streitsache Sexualdelikte" des schleswig-holsteinischen Ministeriums für Soziales, Gesundheit, Familie und Gleichstellung und des Landesverbands Frauenberatung Schleswig-Holstein.

2 Studie „Lebenssituation, Sicherheit und Gesundheit von Frauen in Deutschland", Repräsentative Untersuchung im Auftrag des Bundesministeriums für Familie, Senioren, Frauen und Jugend, durch Prof. Dr. Ursula Müller, Dr. Monika Schröttle, 2004 (Kurzfassung), S. 7.

3 Näheres hierzu: Studie ,Lebenssituation, Sicherheit und Gesundheit von Frauen in Deutschland" (Langfassung), S. 152 f.

$4 \quad 10.891$ aufgeklärte Fälle von Vergewaltigung und Sexueller Nötigung im Jahr 2011 lt. PKS (Polizeiliche Kriminalstatistik) des Bundeskriminalamts <http://www.bka.de/DE/Publikationen/ PolizeilicheKriminalstatistik/pks_node.html> (Zugriff: 17.4.2013) und 1.381 Verurteilungen 2011 It. Verurteiltenstatistik des Statistischen Bundesamts, <https://www.destatis.de/DE/ ZahlenFakten/GesellschaftStaat/Rechtspflege/Strafverfolgung/ Tabellen/VerurteilteStrafart.html> (Zugriff: 17.4.2013).

$5 \quad 807.815$ Verurteilte, 3.276 .901 (54,7\% von 5.990.679) aufgeklärte Straftaten. Die Zahlengrundlage ist den Veröffentlichungen des Statistischen Bundesamts und der Polizeilichen Kriminalstatistik des Bundeskriminalamts entnommen: <https://www.destatis. de/DE/ZahlenFakten/GesellschaftStaat/Rechtspflege/ Strafverfolgung/Tabellen/VerurteilteStrafart.html> (Zugriff: 17.4.2013) und <http://www.bka.de/DE/Publikationen/ PolizeilicheKriminalstatistik/pks_node.html> (Zugriff: 17.4.2013). 